\title{
Perfil epidemiológico e fatores determinantes na saúde ambiental da tuberculose no Brasil
}

A tuberculose é uma doença que assola o mundo desde o início dos tempos, caracterizado por um grave problema de saúde pública. Por se tratar de uma doença infectocontagiosa, a tuberculose tem um grau de transmissão muito eficiente e eficaz, pois o hospedeiro se contamina com os bacilos através das suas vias respiratórias, através de partículas aerossóis contaminados. Fatores ambientais como poluição do ar, diversos tipos de fumaça, podem ser foco para a tuberculose uma vez que esses fatores são veículos para contaminação de pequenas residências, presídios com capacidade de lotação sobrecarregada, com pouca circulação de ar. É uma pesquisa descritiva epidemiológica retroativa, coletada na base de dados SINAN, no Brasil, no ano de 2019. As variáveis avaliadas nos resultados foram: Zona Residência segundo coinfecção Tuberculose/HIV, faixa Etária Segundo Ano Diagnostico, Casos confirmados por Grau de Escolaridade, Casos confirmados por Sexo e Casos confirmados por Forma Clínica. Foram registrados casos de coinfeç̧ão tuberculose/HIV, por Zona urbana, o maior número de casos notificados, com percentual de $72,2 \%(n=6.486)$, a Zona Rural apresentou 4,5\% $(n=411)$, na faixa etária de $20-39$ anos, com o total de $46,10 \%$ ( $n=41.977)$, 0 grau de escolaridade mais acometido foi a de 50 a 8 o serie incompleta do Ensino Fundamental, com percentual de $18,59 \%$ ( $n=16.936)$, homens percentuando $69,90 \%(n=63.653)$, a forma clínica predominante na população Brasileira é a Pulmonar, registrando $84,49 \%$ ( $n=76.941)$. A importância para a cura do paciente consiste no diagnóstico precoce da doença, isso reduz a mortalidade, a criação de novas políticas públicas que visem educar a população, prevenir a doença e promover a saúde, pode erradicar a infecção e salvar futuras gerações.

\section{Epidemiological profile and determinant factors in the environmental health of tuberculosis in Brazil}

The Tuberculosis is a disease that has desolate the world since the beginning of time, characterized by a serious public health problem. Because it is an infectious disease, tuberculosis has a very efficient and effective degree of transmission, as the host is contaminated with the bacilli through its respiratory tract, the selfhood is contaminated by aerosol particles. Environmental factors such as air pollution, a lot of types of smoke, may can be a focus for tuberculosis, once these factors are vehicles for the contamination of small residences, prisons with overloaded capacity, with little air circulation. It is a retrospective epidemiological descriptive research, collected in the SINAN database, in Brazil, in 2019. The variables evaluated in the results were: Residence Zone according to Tuberculosis/HIV coinfection, Age group According to Diagnostic Year, Cases confirmed by Education Level, Cases confirmed by Sex and Cases confirmed by Clinical Form. Tuberculosis/HIV coinfection cases were recorded, by urban area, the highest number of reported cases, with a percentage of $72.2 \%(n=6,486)$, the rural area presented $4.5 \%$ ( $n=$ 411), in the age group $20-39$ years, with a total of $46.10 \%(n=41,977)$, the most affected level of education was the 5 th to 8 th grade of elementary school, with a percentage of $18.59 \%(n=16,936)$, men accounting for $69.90 \%(n=63,653)$, the predominant clinical form in the Brazilian population is the Pulmonary, scoring $84.49 \%(n=76,941)$. The importance for the cure of the patient consists in the early diagnosis of the disease, this reduces mortality, the creation of new public policies to educate the population, prevent the disease and promote health, can eradicate the infection and save future generations.

Keywords: Tuberculosis; Epidemiology; Infection; Public Health.

Topic: Epidemiologia e Saúde Ambiental

Reviewed anonymously in the process of blind peer.
Received: 02/12/2020

Approved: 22/12/2020

\begin{abstract}
Adriano Menino de Macedo Júnior (iD)
Centro Universitário Natalense, Brasil

http://lattes.cnpq.br/4134152465913204

http://orcid.org/0000-0001-6367-1088

adrianomenino2016@gmail.com
\end{abstract}

Clécio Danilo Dias da Silva (iD

Universidade Federal do Rio Grande do Norte, Brasil

http://lattes.cnpq.br/4235157508528733

http://orcid.org/0000-0002-7776-8830

danilodiass18@gmail.com

Elannia Marte de Araújo

Centro Universitário Natalense, Brasil

http://lattes.cnpq.br/3019980760779837

elannia-araujo@hotmail.com

\section{Jhuliete Duarte da Silva \\ Faculdade Venda Nova do Imigrante, Brasi \\ http://lattes.cnpq.br/6186232992315867 drajhuliete@gmail.com}

\section{Josimar Torres Gomes}

Universidade Federal Rural de Pernambuco, Brasi http://lattes.cnpq.br/5974891623513289 adrianomenino2016@gmail.com

\section{Juliane Sibele Cabral Granjeiro}

Faculdade Mauricio de Nassau, Brasil

http://lattes.cnpq.br/1260938967326710

sibelejuliane@gmail.com
Mailda dos Santos Rocha

Centro Universitário Natalense, Brasil http://lattes.cnpq.br/6469527080553505 maildasantos24@gmail.com

\section{Referencing this:}

MACEDO JUNIOR, A. M.; SILVA, C. D. D.; ARAÚJO, E. M.; SILVA, J. D. GOMES, J. T.; GRANJEIRO, J. S. C.; ROCHA, M. S.. Perfil epidemiológico e fatores determinantes na saúde ambiental da tuberculose no Brasil. Revista Ibero Americana de Ciências Ambientais, v.11, n.7, p.243252, 2020. DOI: http://doi.org/10.6008/CBPC21796858.2020 .007 .0022 


\section{INTRODUÇÃO}

A tuberculose é uma doença que assola o mundo desde o início dos tempos, caracterizado por um grave problema de saúde pública (TELES FILHO et al., 2019). A tuberculose é causada pelo agente etiológico Mycobacterium tuberculosis ou Bacilo de koch, trata-se de uma doença infectocontagiosa, grave que muitas vezes leva o paciente a óbito (SANTOS et al., 2019).

A evidências na história de acordo com o trabalho de Cardoso et al. (2018), que a tuberculose se disseminou pelas Américas através dos imigrantes Asiáticos. Possivelmente uma múmia peruana (1.100 a. C.) estaria infectada com a tuberculose, pois houve diagnostico de lesões pulmonares e DNA compatíveis com a M. tuberculosis. A tuberculose atravessa a mais primordial linha do tempo, desde a revolução industrial no século XVIII ao século XIX, na Europa Ocidental que ficou conhecida como peste branca, por sua alta taxa de mortalidade, nesse período a tuberculose estava associada à ambientes de trabalho assustadoramente deterioradas, aposentos impróprios com excesso de contingente e a má circulação de ar puro, dentre outros fatores determinantes favoráveis a doença (MASSABNI et al., 2019).

Por se tratar de uma doença infectocontagiosa, a tuberculose tem um grau de transmissão muito eficiente e eficaz, pois o hospedeiro se contamina com os bacilos através das suas vias respiratórias, espessas no ar, transmitidas pela fala, espirro ou tosse por indivíduos com a doença ativa, a doença pode-se instalar em diversos órgãos do hospedeiro, fatores agravantes podem ser decisivos na vida do paciente, classificandoos como grupo de risco, como a coinfecção pelo vírus do HIV/AIDS, bem como portador de outras doenças crônicas e comorbidades, cepas resistentes a antibióticos disponíveis para o tratamento, o diagnostico tardio, ocasionando a não adesão ao tratamento logo no surgimento, usuários de drogas e cigarros (SANTOS et al., 2020).

A tuberculose está também associada à pobreza extrema, principalmente em lugares remotos do Brasil, com a pobreza se desencadeia diversos outros problemas como a fome, a miséria, desnutrição, condições de higiene escassas, bem como a falta de saneamento básico, políticas públicas criadas atualmente fornecem tratamento gratuito a população, porém há o desafio para que esse tratamento chegue até as mãos dos usuários do Sistema Único de Saúde - SUS (TAVARES et al., 2020).

Diante do atual cenário da Tuberculose, este trabalho tem o objetivo de explicitar o perfil epidemiológico da Tuberculose no Brasil em 2019, bem como o estudo agrega valor para o Sistema de Saúde Pública, para atuar na elaboração de programas educativos de prevenção, bem como alertar os órgãos responsáveis para a elaboração de novos métodos de contenção da doença, ações de vigilância em saúde, garantir adesão ao tratamento da população doente.

\section{REVISÃO TEÓRICA}

\section{Fatores Ambientais que Contribuem para a Infecção por Tuberculose}

É de suma importância que os casos de tuberculose sejam devidamente notificados no Sistema de Informações de Agravos e Notificações - SINAN, através do seu comunicante, a Vigilância Epidemiológica 
deve garantir supervisão dos casos confirmados, garantir adesão ao tratamento disponível, acesso a exames laboratoriais para monitorar a infecção, assim a prevenção e controle da doença em determinados ambientes do país estão asseguradas (SILVA et al., 2019).

A tuberculose pode influenciar diretamente o ambiente social das pessoas infectadas, a doença não traz somente o grave caso clínico, mas também a perturbação social do indivíduo, o preconceito ainda é uma constante variável na sociedade, os portadores da infecção são levados a se afastarem de seus entes queridos bem como cumprir isolamento social (SILVA et al., 2019).

Diversas infecções graves que assolam diversos países estão diretamente relacionadas às degradações ambientais, regiões em desenvolvimento urbanístico tende a impactar a vida e saúde da população que reside naquele determinado ambiente. Os fatores ambientais tornam a tuberculose um problema à saúde pública, uma vez que acometem mais países em desenvolvimento (FERNANDES, 2018; TEMOTEO et al., 2019).

Fatores ambientais como poluição do ar, diversos tipos de fumaça, podem ser foco para a tuberculose, uma vez que esses fatores são veículos para contaminação de pequenas residências, presídios com capacidade de lotação sobrecarregada, com pouca circulação de ar (FERNANDES, 2018; PELISSARI, 2019).

Condições ambientais desfavoráveis, tais como, a pobreza extrema, falta de saneamento básico, moradia impropria, são marcadores para casos positivos da tuberculose. No que tange a distribuição de recursos em saúde per capita desordenada, pode comprometer a saúde da população de um determinado país, desprovendo serviços sanitários obrigatórios, vigilância em saúde, dificultando o diagnóstico precoce da tuberculose (FERNANDES, 2018; BUSATTO et al., 2020).

Outro fator ambiental que pode estar relacionado à tuberculose, é a sazonalidade, a doença pode ter maior índice de transmissão em determinadas épocas do ano, no inverno, por exemplo, a população tende a ficar em ambientes fechados e aglomerados, contribuindo para a o aumento da transmissão. Ambientes com Superfície úmida podem garantir a sobrevida de micobactérias por até 43 dias, o que favorece a contaminação de pessoas, por tuberculose. Ambientes que favorecem correntes de ar, luz ultravioleta e outros tipos de radiação podem desativar os bacilos (FERNANDES, 2018; TITOSSE, 2019).

\section{Aspectos epidemiológicos da tuberculose em nível global e no Brasil}

A epidemiologia é a ciência que estuda o mecanismo de ação de cada doença, ou seja, sua transmissibilidade, sua frequência, seu modo de distribuição ao redor do mundo, sua evolução, as epidemiologias também criam medidas educativas para a prevenção de doenças (BASSANEZI et al., 2019).

Do ponto de vista epidemiológico a tuberculose pode ser contida e erradicada nos pacientes, quando esta é diagnosticada precocemente, e quando a adesão ao tratamento é de $100 \%$, isso afeta diretamente na erradicação da reincidência da tuberculose, a sua propagação e a sua alta taxa de mortalidade. A tuberculose prevalece em países subdesenvolvidos ou em fase de crescimento urbanístico ou econômico, ou seja, o fator da economia interfere diretamente nas altas taxas de contaminação pela tuberculose (FORTUNA et al., 2020). 
Em 2010 o Brasil notificou 85 mil novos casos de tuberculose, os óbitos chegaram a cinco mil no mesmo ano, só na Região Nordeste os casos de tuberculose chegaram a 19.589 novos casos confirmados, destacando-se a segunda região do país, mais acometida pela doença, em 2015 a doença contaminou 10,4 milhões da população mundial, desse total 1,4 milhões da população foi a óbito (FORTUNA et al., 2020).

De acordo com o estudo de Silva et al. (2019) somente no ano de 2011, o Brasil registrou 69.245 casos de tuberculose positivo, valor esse referente a cada 100.000 habitantes, esse valor foi bem distribuído por todas as Regiões do país, sendo na Região Norte com 45,2\%, Região Nordeste com 35,9\%, Região Sudeste com 37,6\%, Região Sul com 33\% e Região Centro-Oeste com $22 \%$ casos confirmados.

De acordo com Silva et al. (2020), a tuberculose acometeu 10 milhões de pessoas a nível global, todos os novos casos foram devidamente registrados, e 1,3 milhões de pessoa vieram a óbito em todo o mundo, no Brasil, houve 69.569 novos casos de tuberculose ainda no ano de 2017, equivalendo um percentual de 33,5 novos casos a cada 100 mil habitantes. Em 2016 o Brasil registrou um número de obituário equivalente a 2,1 óbitos a cada 100 mil habitantes, com total de 4.426 casos de óbitos notificados.

O problema da não notificação da tuberculose está na rotatividade dos colaboradores das Unidades de Saúde, o diagnostico tardio da doença, implicam para a subnotificação dos casso da tuberculose, a falta de informação corrobora para o atraso no diagnóstico precoce e na adesão ao tratamento quimioterápico (SILVA et al., 2020).

\section{MATERIAIS E MÉTODOS}

Esta pesquisa é descritiva, retrospectiva epidemiológica, realizado por meio da fonte de dados disponibilizados no DATASUS, através do Sistema de Informação de Agravos de Notificação - SINAN, no Brasil - BR. A coleta de dados ocorreu em Julho de 2020, e trata-se de notificações compulsórias datadas em 2019.

De acordo com o Ministério da Saúde, o SINAN, é sustentado, primordialmente, pelas notificações compulsórias, que são registradas com base no registro, investigação e diagnósticos das doenças, que constam na lista nacional de doenças de notificação compulsória.

As variáveis avaliadas nos resultados foram: Zona Residência segundo coinfecção Tuberculose/HIV, faixa Etária Segundo Ano Diagnostico, Casos confirmados por Grau de Escolaridade, Casos confirmados por Sexo e Casos confirmados por Forma Clínica.

Todos os dados epidemiológicos coletados para análise foram selecionados e obtidos por meio do aplicativo TABNET, a partir de suas caixas de opções (linha, coluna e conteúdo). Por se tratar de dados secundários de um banco de dados de domínio público, não foi necessário submeter o trabalho a apreciação do Comitê de Ética em Pesquisa em Seres Humanos (CEP), além disso, os dados são secundários e anônimos.

A metodologia da presente pesquisa utilizou também pesquisas bibliográficas, como revisão de literatura, artigos originais de pesquisas, a qual foi lastreada em artigos constantes na base eletrônica SciELO, Google acadêmico, Pubmed, MEDLINE (National Library of Medicine, USA - NLM), assim como repositórios de universidades públicas e privadas, empregando-se as palavras chave: Tuberculose, Epidemiologia, infecção, Saúde Pública. 
A pesquisa considerou artigos publicados entre os anos de 2018 a 2020, de forma que se realizou, inicialmente, a seleção, por vias das palavras chaves. Em seguida, promoveu-se o fichamento do material selecionado e elegeram-se os artigos que faziam alusão ao tema. Os critérios de inclusão foram: artigos correlatos ao tema, publicados entre 2018 e 2020, dentro da área de saúde pública.

\section{RESULTADOS E DISCUSSÃO}

Em 2019, o Brasil registrou 91.056 novos casos de tuberculose, todos os casos devidamente documentados junto à lista Nacional de Doenças de Notificações Compulsórias, e disponibilizados através do SINAN. No Brasil a tuberculose acomete principalmente pessoas portadoras do Vírus da Imunodeficiência Humana (HIV), devido a sua condição, no qual se encontra com o sistema imunológico em depressão, o vírus atinge em particular as células T CD4+, destruindo esses linfócitos e contaminando novas células de defesa (PATEL et al., 2018).

Neste estudo, em 2019 os dados apontam que foram confirmados $9.85 \%$ ( $n=8.972$ ) novos casos positivos de coinfecção de Tuberculose e HIV, ver tabela 1, sendo na Zona urbana, o maior número de casos notificados, com percentual de $72,2 \%(n=6.486)$ novos casos confirmados, a Zona Rural apresentou $4,5 \%$ ( $n=$ 411) novos casos confirmados. Em contrapartida podemos ver que, $66,82 \%$ ( $n=60.847)$ da pulação infectada com Tuberculose não tiveram coinfecção com o vírus do HIV.

A coinfecção de TB/HIV pode ocorrer em lugares com elevadas taxas de pobreza e desigualdade econômica, atingindo populações menos escolarizadas, esse fator pode mudar drasticamente a vida emocional dos pacientes bem como impactar a sua situação econômica (MIRANDA et al., 2018).

Tabela 1: Casos confirmados por Zona Residência, segundo coinfecção Tuberculose/HIV. Brasil, 2019.

\begin{tabular}{l|l|l|l|l|l}
\hline HIV & Ign./Branco & Urbana & Rural & Periurbana & Total \\
\hline Em Branco/IGN & 771 & 41 & 17 & - & 829 \\
\hline Positivo & 2.008 & 6.486 & 411 & 67 & 8.972 \\
\hline Negativo & 16.353 & 38.575 & 5.480 & 439 & 60.847 \\
\hline Em andamento & 1.224 & 4.386 & 438 & 33 & 6.081 \\
\hline Não realizado & 2.332 & 10.685 & 1.208 & 102 & 14.327 \\
\hline Total & 22.668 & 60.173 & 7.554 & 641 & 91.056 \\
\hline
\end{tabular}

Fonte: BRASIL (2007).

Consoante Rossetto et al. (2019) destacam em sua pesquisa realizada na cidade do Porto Alegre, o registro de 2.419 casos de coinfecção de tuberculose e HIV, no período de 2009 a 2013, desse total, as unidades de saúde da cidade como as Unidades de Administração Distrital - DAUs e Lomba do Pinheiro (PLP), apresentaram maior casos de coinfecção, com 557 casos confirmados.

Segundo Miranda et al. (2018), em seu estudo epidemiológico, realizado com dados de 558 microrregiões do Brasil, foi constatado 47.265 casos de coinfecção de Tuberculose e HIV/Aids, sendo que apenas 33.773 do total de casos analisados tratavam-se de novos casos de coinfecção, o restante não se aplicava.

O estudo epidemiológico de Oliveira et al. (2018), realizado no Estado do Piauí, descreveu que dos 10.154 casos notificados de Tuberculose, apenas $6 \%(n=619)$ correspondiam a casos de coinfecção de 
Tuberculose e HIV/Aids.

A pesquisa descritiva e ecológica realizada por Cavalin (2018), no munícipio de São Paulo no período de 2007 até 2015, registra que a coinfecção Tuberculose e HIV/AIDS, em características sociodemográficas que os pacientes que tinham residência fixa apresentaram um total de 5.609 casos, enquanto os pacientes que não tinham residência fixa apresentaram um total de 483 casos confirmados.

A Tuberculose pode incidir diretamente de acordo na faixa etária da pessoa, fatores esses que podem fazer com que o indivíduo adquira a doença após sua infecção. Pessoas com idades avançadas são mais propensas a contrair a Tuberculose, enquanto que, adolescentes e adultos jovens, podem desenvolver a doença ao longo prazo, com período de incubação e latência maior do que o normal (SILVEIRA, 2019; SANTOS et al., 2018).

O fator da faixa etária também está relacionado diretamente à situação financeira de determinada Região ou País, pois em regiões ou países mais desenvolvidos, o público alvo é idoso, esse grupo é caracterizado como de risco, a supressão do sistema imunológico pode ocasionar a reativação das cepas de tuberculose latente, enquanto que em países ou Regiões menos desenvolvidas o público mais atingido pela tuberculose são os indivíduos mais jovens (SILVEIRA, 2019; SANTOS et al., 2018).

Este estudo apresenta um número elevado de casos confirmados de Tuberculose, na faixa etária de 20 - 39 anos, com o total de 46,10\% ( $n=41.977)$, e na faixa etária de 40 - 59 também apresentou um índice muito elevado de casos confirmados de Tuberculose com 31\% ( $n=27.936)$, em 2019 (ver tabela 2). Essa faixa etária é muito acometida por infecções recentes de Tuberculose, isso pode se dar porque o Brasil tem um aumento crescente na transição da população adulta para a idosa, o que concerne o aumento das infecções.

Tabela 2: Casos confirmados por Faixa Etária, Segundo Ano Diagnostico. Brasil, 2019.

\begin{tabular}{l|l}
\hline Faixa Etária & Total \\
\hline Em branco/IGN & 21 \\
\hline$<1$ ano & 543 \\
\hline $1-4$ & 648 \\
\hline $5-9$ & 625 \\
\hline $10-14$ & 999 \\
\hline $15-19$ & 4.897 \\
\hline $40-59$ & 41.977 \\
\hline $60-64$ & 27.936 \\
\hline $65-69$ & 4.714 \\
\hline $70-79$ & 3.371 \\
\hline 80 e + & 3.767 \\
\hline Total & 1.554 \\
\hline Fonte: BRASIL 207$)$ & 91.056 \\
\hline
\end{tabular}

Fonte: BRASIL (2007).

Os dados da variável faixa etária corroboram com o estudo de Macedo et al. (2018), onde dos 570 casos de Tuberculose, prevalente na faixa etária de $20-39$ anos, totalizando $42,3 \%$ ( $n=241$ ), em cima do total de casos.

Os achados de Pereira et al. (2018), também argumentam com o presente estudo, em sua pesquisa realizada no Estado do Rio de Janeiro, foram analisados 670 casos de Tuberculose, onde desse total a faixa etária mais acometida foi a de $30-39$ anos com percentual de $23 \%$ ( $n=154)$. 
A infecção causada pela Tuberculose pode atingir todas as classes sociais, dentro deste contexto podemos ver que a infecção pode atingir sobre tudo as populações menos escolarizadas, pois são essas pessoas que estão carentes de conhecimentos sobre o agente etiológico causador da doença, bem como a falta do conhecimento de como se prevenir e se cuidar (SANTOS et al., 2020).

A partir da atual pesquisa podemos ver que dos 91.056 casos de Tuberculose diagnosticados, o grau de escolaridade mais acometido foi a de 50 a 8 o serie incompleta do Ensino Fundamental, com percentual de 18\% ( $n=16.936)$, e a população com grau de escolaridade Ensino Médio Incompleto, com percentual de 12\% ( $n=11.340)$ (Ver Gráfico 1).

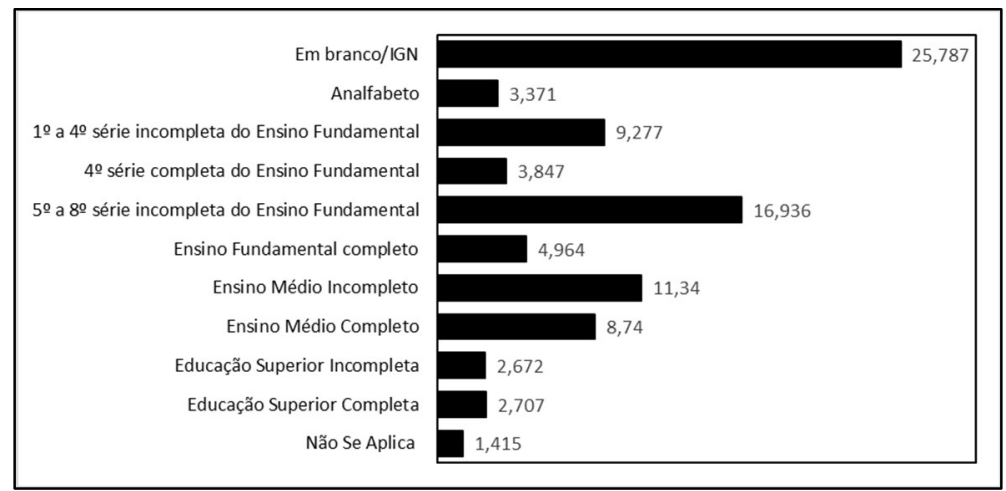

Gráfico 1: Casos confirmados por Grau de Escolaridade, Segundo Ano Diagnostico. Brasil, 2019. Fonte: BRASIL (2007).

Os resultados desta pesquisa corroboram com os achados de Fontes et al. (2019), realizado no Brasil - BR, no período de 2012 a 2016, onde destaca que as pessoas mais infectadas pela Tuberculose apresentavam analfabetismo ou baixa escolaridade, os homens analfabetos representaram o maior número correspondendo a 231.186 número de casos confirmados.

Outro estudo que corrobora com esta pesquisa é o de Santos et al. (2018), realizado no Estado da Bahia - Salvador, que verificou que no período de 2006 a 2015, a população com mais infectado pela Tuberculose foi a que dispunha apenas do Ensino Fundamental Incompleto, registrando um total de 54,6\% $(n=879)$, valor esse do referente ao total de casos investigado no período supracitado, que foi 1.611 casos.

Geralmente os homens estão mais suscetíveis à infecção da Tuberculose, por estarem mais vulneráveis a Silicose, abuso de drogas, coinfecções com outras doenças respiratórias e HIV/AIDS, isso tudo pode agravar o sistema imune dos pacientes (SANTOS et al., 2018).

Nesta pesquisa destacamos que a infecção da Tuberculose atinge principalmente homens percentuando $70 \%$ ( $n=63.653$ ) casos confirmados, enquanto que as mulheres apresentam $30 \%(n=27.393)$ (Gráfico 2).

A variável aqui explicita correspondem com os achados de Moraes et al. (2018), em seu trabalho realizado no Estado do Maranhão em 2018, o sexo masculino foi mais acometido pela Tuberculose com 32 casos confirmados, enquanto que o sexo feminino apresentou apenas 24 casos confirmados.

O estudo de Santos et al. (2019), constatou que dos casos analisados do obituário em um munícipio específico no Sul do Mato Grosso, 25 eram do sexo masculino enquanto que o sexo feminino apenas 7 foram a óbito, isso se deve por que os homens tendem a buscar as unidades de saúde tarde, interferindo em um 
diagnóstico precoce.

A tuberculose pode ocorrer em várias formas clínicas, o agente etiológico tem maior interesse nos pulmões isso por que esses órgãos armazenam grande quantidade de oxigênio, o qual favorece a proliferação do M. Tuberculoses, que é uma bactéria exclusivamente aeróbica (CARDOSO et al. 2018).

No Gráfico 3, podemos ver as formas clínicas diagnosticadas nos pacientes. A forma clínica predominante na população Brasileira é a Pulmonar, registrando $84 \%$ ( $n=76.941)$ casos confirmados, seguida da forma extrapulmonar com $12 \%$ ( $n=11.523)$, e, pulmonar+extrapulmonar com $1 \%(n=2.525)$ dos casos confirmados.

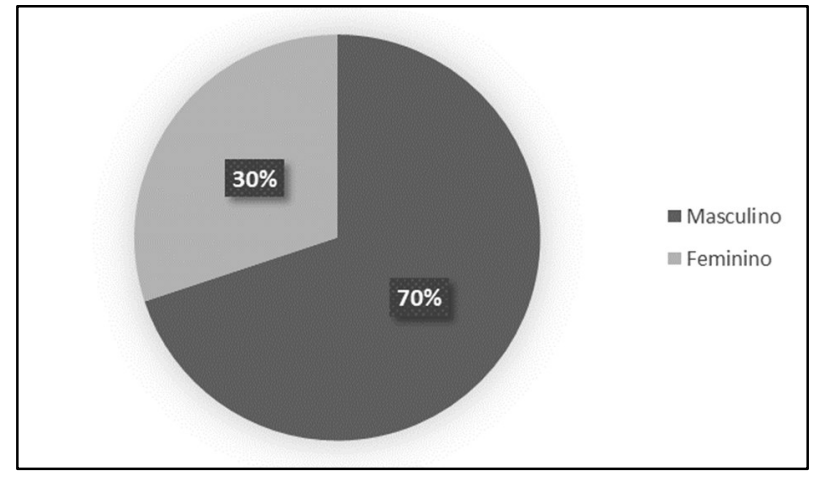

Gráfico 2: Casos confirmados por Sexo, Segundo Ano Diagnostico. Brasil, 2019.

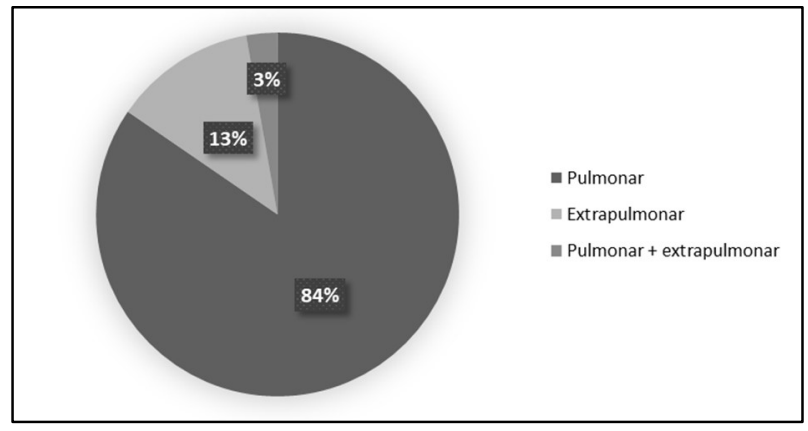

Gráfico 3: Casos confirmados por Forma Clínica, Segundo Ano Diagnostico. Brasil, 2019.

Fonte: BRASIL (2007).

A pesquisa que corrobora com este estudo é o de Cardoso et al. (2018), na Microrregião de Umuarana, onde dos 533 casos confirmados de tuberculose, 435 eram na forma clínica pulmonar, e a segunda forma que também mais acometeu foi o da extrapulmonar com 80 casos confirmados de tuberculose.

Consoante Neves et al. (2018), em sua pesquisa realizada no Estado do Pará, avaliou 40.377 prontuários de pacientes com Tuberculose confirmada, desse total $87,3 \%(n=31.372)$ foram acometidos pela forma clínica pulmonar, e 11,9\% ( $n=2.182)$ eram na forma extrapulmonar.

\section{CONCLUSÕES}

Está pesquisa revela o perfil epidemiológico da Tuberculose em todo o Brasil no ano de 2019. A tuberculose é uma doença grave que apresenta um problema de Saúde pública em todo o mundo. A adesão ao tratamento completo dos antimicrobianos e antibacterianos podem salvar vidas, e melhora da qualidade de vida das pessoas.

A tuberculose é uma doença infectocontagiosa, que apesar da sua alta taxa de mortalidade, o Sistema Único de Saúde, fornece tratamento eficaz e eficiente, curando o paciente acometido. A importância para a cura do paciente consiste no diagnóstico precoce da doença, isso reduz a sua mortalidade, a criação de novas políticas públicas que visem educar a população, prevenir a doença e promover a saúde, pode erradicar a infecção e assegurar futuras gerações.

Ter o conhecimento absoluto do perfil epidemiológico da tuberculose no Brasil contribui para um 
melhor entendimento de como a doença pode atingir as diversas regiões do país, por meio desta pesquisa, autoridades competentes podem elaborar estratégias, que atuam na Atenção Básica de Saúde, garantindo tratamento disponível ao alcance de todos, bem como salvar a vida das populações mais vulneráveis e susceptíveis à doença.

\section{REFERÊNCIAS}

BASSANEZI, R. B.; LOPES, S. A.; JÚNIOR, J. B.; SPÓSITO, M. B.; YAMAMOTO, P. T.; MIRANDA, M. P.; WULFF, N. A.. Epidemiologia do huanglongbing e suas implicações para o manejo da doença. Citrus Research \& Technology, v.31, n.1, p.11-23, 2019.

BRASIL. Ministério da Saúde. Secretaria de Vigilância em Saúde. Departamento de Vigilância Epidemiológica. Sistema de Informação de Agravos de Notificação - SINAN: normas e rotinas. Brasília: Ministério da Saúde, 2007.

BUSATTO, C.; VIANNA, J. S.; SILVA, A. B. S.; BASSO, R.; SILVEIRA, J.; GROLL, A. V.; SILVA, P. E. A. D.. Micobactérias não tuberculosas em pacientes com suspeita de tuberculose e a diversidade genética de Mycobacterium avium no extremo sul do Brasil. Jornal Brasileiro de

Pneumologia, v.46, n.2, 2020. DOI:

https://doi.org/10.36416/1806-3756/e20190184

CARDOSO, B. A.; MARTINS, A. C. G. S.; DIAS, G. A. S.; MORAES NETO, A. H. A.; SAAD, M. H. F.. Populações negligenciadas e fatores socioeducacionais: Aplicação de um guia metodológico para tuberculose e parasitoses intestinais. Revista Ciência \& Saberes-UniFacema, p.970977, 2018.

CARDOSO, L. C.; MELQUIADES, R.; MAGALHÃES, J. A.; CARVALHO, S. S.; ROSA, G.; MERLINI, L. S.; JULIÃO, V. A. P.. Aspectos epidemiológicos dos pacientes notificados com tuberculose na microrregião de Umuarama-noroeste paranaense de 2009 a 2014. Arquivos de Ciências da Saúde da UNIPAR, v.22, n.3, 2018. DOI:

https://doi.org/10.25110/arqsaude.v22i3.2018.6162

CAVALIN, R. F.. Coinfecção TB-HIV: análise espacial e temporal no município de São Paulo. Dissertação (Mestrado em Ciências) - Universidade de São Paulo, São Paulo, 2018. DOI: https://doi.org/10.11606/D.6.2018.tde-11062018$\underline{095251}$

FERNANDES, F. M. D. C.. Análise da incidência de tuberculose em três municípios brasileiros e o Distrito Federal na perspectiva dos fatores climáticos e sociais. Tese (Doutorado em Enfermagem) - Universidade de Brasília, Brasília, 2018.

FONTES, G. J. F.; SILVA, T. G.; SOUSA, J. C. M.; FEITOSA, A. D. N. A.; SILVA, M. L.; BEZERRA, A. L. D.; ASSIS, E. V.. Perfil Epidemiológico da Tuberculose no Brasil no Período de 2012 a 2016. Revista Brasileira de Educação e Saúde, v.9, n.1, p.19-26, 2019. DOI: https://doi.org/10.18378/rebes.v9i1.6376

FORTUNA, J. L.; SOARES, P. A. O.. Tuberculosis epidemiological profile in Teixeira de Freitas municipality from 2001 to 2017. Brazilian Journal of Health Review, v.3, n.3, p.7171-7192, 2020. DOI:

https://doi.org/10.34119/bjhrv3n3-247

MACEDO, J. L.; OLIVEIRA, A. S. D. S. S.; PEREIRA, I. C.; ASSUNÇÃO, M. D. J. S. M.. Perfil epidemiológico da tuberculose em um Município do Maranhão. Revista Ciência \& Saberes-UniFacema, v.3, n.4, p.699-705, 2018.

MASSABNI, A. C.; BONINI, E. H.. Tuberculose: história e evolução dos tratamentos da doença. Revista Brasileira Multidisciplinar, v.22, n.2, p.6-34, 2019. DOI:

https://doi.org/10.25061/25272675/ReBraM/2019.v22i2.678

MORAES, M. F. V.; CORRÊA, R. D. G. C. F.; COUTINHO, N. P. S.; CALDAS, A. D. J. M.; SILVA, T. C.; SANTOS, K. C. B.; AQUINO, D. M. C.. Perfil epidemiológico de casos de tuberculose em um município prioritário no estado do Maranhão. Revista de Pesquisa em Saúde, v.18, n.3, 2018.

NEVES, D. C. D. O.; LOUREIRO, L. D. O.; PAIVA, N. P.; OHNISHI, M. D. D. O.; RIBEIRO, C. D. T.. Aspectos epidemiológicos da tuberculose nas Regiões de Integração do estado do Pará, Brasil, no período entre 2005 e 2014. Revista Pan-Amazônica de Saúde, v.9, n.3, p.21-29, 2018. DOI: http://dx.doi.org/10.5123/s217662232018000300003

OLIVEIRA, L. B.; COSTAR, C. R. B.; QUEIROZ, A. A. F. L. N.; ARAÚJO, T. M. E.; SOUSA, K. A. A.; REIS, R. K.. Análise epidemiológica da Coinfecção Tuberculose/HIV. Cogitare Enfermagem, v.23, n.1, 2018. DOI: http://dx.doi.org/10.5380/ce.v23i1.51016

PATEL, P.; ROSE, C. E.; COLLINS, P. Y.; NUCHE-BERENGUER, B.; SAHASRABUDDHE, V. V.; PEPRAH, E.; HIV, N.. Doenças não transmissíveis entre pessoas infectadas pelo HIV em países de baixa e média renda: uma revisão sistemática e metanálise. AIDS, n.24, p.123-137, 2010. DOI: http://dx.doi.org/10.1097/QAD.0000000000001888

PELISSARI, D. M.. Impacto do encarceramento na incidência da tuberculose. Tese (Doutorado em Ciências) Universidade de São Paulo, São Paulo, 2019. DOI: https://doi.org/10.11606/T.6.2019.tde-11062019-140751

PEREIRA, A. G. L.; ESCOSTEGUY, C. C.; GONÇALVES, J. B.; MARQUES, M. R. V. E.; BRASIL, C. M.; SILVA, M. C. S.. Fatores associados ao óbito e ao abandono do tratamento da tuberculose em um hospital geral do município do Rio de Janeiro, 2007 a 2014. Rev. Epidemiol. Control. Infec., v.8, n.2, p.150-158, 2018.

ROSSETTO, M. M.; BRAND, E. M. M. B.; HAHN G. V.; OLIVEIRA D. L. L. C.; TEIXEIRAL, B.. Perfil epidemiológico dos casos de tuberculose com coinfecção pelo HIV na cidade de Porto Alegre, Brasil. Revista Brasileira de Enfermagem, v.72, 
n.5, p.1211-1218, 2019. DOI: https://doi.org/10.1590/0034$\underline{7167-2017-0613}$

SANTOS, D. A. S.; MARQUES, A. L. A.; GOULART, L. S.; OLINDA, R. A.. Fatores associados aos óbitos hospitalares por tuberculose em um município no sul de Mato Grosso, 2008-2017. Multitemas, v.24, n.56, p.135-150, 2019. DOI: https://doi.org/10.20435/multi.v24i56.2031

SANTOS, Á. N.; SANTOS, M. R.; GONÇALVES, L. V. P.. Perfil epidemiológico da tuberculose em uma microrregião da Bahia (2008-2018). Revista Brasileira de Saúde Funcional, v.10, n.1, p.29-29, 2020.

SANTOS, J. G. C.; ROCHA, M. A. D. N.; SANTOS, R. C.; RIBAS, J. L. C.. Perfil clínico e epidemiológico da tuberculose em Alagoas de 2008 a 2017. Revista Saúde e Desenvolvimento, v.13, n.14, p.35-48, 2019.

SANTOS, J. N. D.; SALES, C. M. M.; PRADO, T. N. D.; MACIEL, E. L.. Fatores associados à cura no tratamento da tuberculose no estado do Rio de Janeiro, 20112014. Epidemiologia e Serviços de Saúde, v.27, e2017464, 2018. DOI: https://doi.org/10.5123/S1679$\underline{49742018000300015}$

SANTOS, M. B. F.; LOURENÇÃO, L. G.; BAPTISTA, M. A.; GAZETTA, C. E.. Perfil da população notificada por tuberculose em um hospital escola do interior paulista de 2010 a 2014. Enfermagem Brasil, v.17, n.1, p.18-25, 2018. DOI: http://dx.doi.org/10.33233/eb.v17i1.1230

SANTOS, S. M.; SANTOS, A. L. V.; FREITAS, B. Q.; MARINS, C. M. F.; CARNICEL, C.; SCHERER, E. F.; DELMONDES, P. H.. Perfil dos pacientes portadores de tuberculose e os fatores de risco associados em municípios da Amazônia legal. Revista Eletrônica Acervo Saúde, v.43, e2344-e2344, 2020. DOI: https://doi.org/10.25248/reas.e2344.2020

SANTOS, T. A. D.; MARTINS, M. M. F.. Perfil dos casos de reingresso após abandono do tratamento da tuberculose em Salvador, Bahia, Brasil. Cadernos Saúde Coletiva, v.26, n.3, p.233-240, 2018. DOI: http://dx.doi.org/10.1590/1414$\underline{462 \times 201800030235}$
SILVA, G. D. M. D.; DUARTE, E. C.; CRUZ, O. G.; GARCIA, L. P.. Identificação de microrregiões com subnotificação de casos de tuberculose no Brasil, 2012 a 2014. Epidemiologia e Serviços de Saúde, v.29, e2018485. 2020. DOI: https://doi.org/10.5123/S1679-49742020000100025

SILVA, P. L. N.; OLIVEIRA, M. K. S.; GUIMARÃES, C. F.; GUIMARÃES, L. F.; SANTOS, L. R.; ALVES, E. C. S.. Análise do conhecimento de moradores quanto à transmissibilidade $\mathrm{e}$ prevenção da tuberculose: implicações biopsicossociais. Journal of Management \& Primary Health Care, v.10, n.3, p.1-16, 2019. DOI: https://doi.org/10.14295/jmphc.v10i0.515

SILVEIRA, O. S.. Análise da distribuição geográfica dos casos de tuberculose e a interação com o ambiente no município do Guarujá. Dissertação (Mestrado em Saúde Coletiva) Universidade Católica de Santos, São Paulo, 2019.

TAVARES, C. M.; CUNHA, A. M. S. D.; GOMES, N. M. C.; LIMA, A. B. D. A.; SANTOS, I. M. R. D.; ACÁCIO, M. D. S.; SOUZA, C. D. F. D.. Tendência e caracterização epidemiológica da tuberculose em Alagoas, 2007-2016. Cadernos Saúde Coletiva, v.28, n.1, p.107-115, 2020. DOI: https://doi.org/10.1590/1414-462x202028010381

TELES FILHO, R. V.; MATOS A. B. E. G.; AZEVÊDO, L. H. S.; MELO, N. C.; RABAHI, M. F.; DAHER, M. T.. Perfil epidemiológico da tuberculose óssea no Brasil, 2012017. Revista de Medicina, v.98, n.5, p.315-323, 2019. DOI: https://doi.org/10.11606/issn.1679-9836.v98i5p315$\underline{323}$

TEMOTEO, R. C. D. A.; CARVALHO, J. B. L. D.; LIRA, A. L. B. D. C.; LIMA, M. A. D.; SOUSA, Y. G. D.. Enfermagem na adesão ao tratamento da tuberculose e tecnologias em saúde no contexto da atenção primária. Escola Anna Nery, v.23, n.3, 2019. DOI: https://doi.org/10.1590/2177-9465-ean-2018$\underline{0321}$

TITOSSE, A. C.. Distribuição espaço-temporal e demográfica dos casos notificados de tuberculose em Maputo-MZ de 2011 a 2016. Dissertação (Mestrado em Meio Ambiente e Desenvolvimento Regional) - Universidade do Oeste Paulista, São Paulo, 2019.

A CBPC - Companhia Brasileira de Produção Científica (CNPJ: 11.221.422/0001-03) detém os direitos materiais desta publicação. Os direitos referem-se à publicação do trabalho em qualquer parte do mundo, incluindo os direitos às renovações, expansões e disseminações da contribuição, bem como outros direitos subsidiários. Todos os trabalhos publicados eletronicamente poderão posteriormente ser publicados em coletâneas impressas sob coordenação da Sustenere Publishing, da Companhia Brasileira de Produção Científica e seus parceiros autorizados. Os (as) autores (as) preservam os direitos autorais, mas não têm permissão para a publicação da contribuição em outro meio, impresso ou digital, em português ou em tradução. 